BNL-61617

March 1995

\title{
Spurious Anomalies in Dimensional Renormalization
}

\author{
T.L. Trueman 冈 \\ Physics Department, Brookhaven National Laboratory, Upton, NY 11973
}

\begin{abstract}
A set of procedures is given for avoiding the spurious anomalies that are generated when the 't Hooft - Veltman definition of $\gamma_{5}$ is used in conjunction with renormalization by minimal subtraction. These procedures are derived from the standard procedure, which requires in addition various finite renormalizations to remove spurious violations of chiral symmetry. They apply to open fermion lines, including flavor changing currents, to closed fermion loops, including those which contain true anomalous currents, and to anomalous loops connected to open fermion lines, to all orders in QCD.
\end{abstract}

\footnotetext{
${ }^{*}$ This manuscript has been authored under contract number DE-AC02-76CH00016 with the U.S. Department of Energy. Accordingly, the U.S. Government retains a non-exclusive, royalty-free license to publish or reproduce the published form of this contribution, or allow others to do so, for U.S. Government purposes.
} 


\section{Introduction}

Since 1972 when 't Hooft and Veltman [1] and others [2] introduced the dimensional regularization of Feynman diagrams the technique has come to play a central role in perturbative field theory calculations. Coupled with the dimensional renormalization procedure [3] it has led to a relatively simple and systematic way of removing the divergences that inevitably accompany such calculations and of obtaining precise predictions for experimental quantities in terms of well-defined theoretical quantities like coupling constants. In their original paper 't Hooft and Veltman recognized the special character of $\gamma_{5}$ : it is an intrinsically fourdimensional object, not zero-dimensional as habit often leads us to assume. In that paper they gave a prescription for handling $\gamma_{5}$ in any dimension; namely

$$
\gamma_{5}=i \gamma_{0} \gamma_{1} \gamma_{2} \gamma_{3}
$$

(This is often refered to as the HV prescription for short.) Although this prescription has been very much used and studied and generally shown to be consistent [4] [5], it has nevertheless proved vexing over the years and continues to do so. This definition treats the first four dimensions of space-time asymmetrically from the rest so that

$$
\begin{gathered}
\left\{\gamma_{5}, \gamma_{\mu}\right\}=0 \quad \text { for } \mu=0,1,2,3 \\
{\left[\gamma_{5}, \gamma_{\mu}\right]=0 \quad \text { otherwise. }}
\end{gathered}
$$

This leads to a certain amount of algebraic complication when compared with the "naive" $\left\{\gamma_{5}, \gamma_{\mu}\right\}=0$ for all $\mu$. It turns out that in very many cases the same result is obtained by either method, so people naturally prefer to be naive. However, it was shown already in [1] that this is not possible for the AVV triangle graph and, indeed it is possible, using techniques subsequently developed, to show that the naive commutation relations would lead to all loops, even convergent ones, containing an odd numbers of $\gamma_{5}$ 's vanishing [4]. This has created the awkward situation where often the naive relations are used except when they are known to give inconsistent results.

A much more serious problem is connected with the violation of chiral symmetry. Again in [1] it was noted that the prescription Eq.2 gave the correct result for the axial anomaly, something that could not occur with a chirally-symmetric regulator. Since this is 
a true anomaly, this is all to the good. However, in addition to this "good" anomaly this regulator also produces a number of "bad", spurious anomalies. These are spurious in the sense that they can be removed by appropriate renormalization. The appropriate renormalization requires renormalization constants which themselves are not chirally symmetric. This was shown long ago in [6] and is systematically discussed in Collin's book, [5]. There is no fundamental problem with the procedure, but it is often very laborious. What makes the situation especially treacherous is when this is encountered in conjunction with dimensional renormalization. This will in general not provide the appropriate renormalization required. The additional finite renormalizations must be put in explicitly "by hand", and they are sometimes overlooked. This danger also arises in calculations which are finite for some algebraic reason. An example of this will be given later.

In this regard, it is important to note the limitations of the oft cited work of Breitenlohner and Maison [4]. That paper carries through very systematically the task of showing that dimensional renormalization is a consistent renormalization procedure. They emphsize, however, that the procedure does not preserve equations of motion and Ward identities when $\gamma_{5}$ is present. See the discussion they give of their Theorem 2 which is limited to the case where there is no explicit dependence in the relations on the dimension $d$. Such $d$-dependence, as pointed out by them, is the source of anomalies. See Bonneau [7] for a thorough discussion of this point. (Bonneau's review also cites a large number of works on this topic. We do not attempt a historical review here, but cite only those papers which directly bear on the present work.)

In most cases these spurious anomalies can be avoided by using the naive commutation relations. This was realized in some of the earliest applications of the method, [8, 9, 10, 11], and led to a series of attempts to formulate rules for manipulating $\gamma_{5}$ in arbitrary dimension that essentially produce the naive rules except in those instances where they are known to be inconsistent and for which appropriate modifications are provided. For one reason or another none of these prescriptions have stuck. We do not propose to review them here, but there are a number of reasons that may explain this. Some sets of rules are not complete or, at least, it is not clear how they are to be applied in general. In other cases, the rules require manipulation of admittedly non-existent objects called $\gamma_{5}$ or they require radical redefinition 
of the operation of taking the trace of a product of matrices around a closed loop.

An instance of this latter approch is to be found in [12]. This is cited here because it was developed in response to a very interesting example of the dangers of problems occuring in finite calculations mentioned above, and because it illustrates the continuing difficulties in handling $\gamma_{5}$ [13]. In that case, the flavor changing decay $Z \rightarrow b+\bar{s}$ is calculated at one loop order. Because of the basic structure of the Lagrangian this must come out to be a finite, well-defined number. The authors of [13] compared the results of using Eq.2 with the results of naively commuting $\gamma_{5}$ and they found that they gave finite but different results. In order to resolve this unsatisfaactory state of affairs they checked the Ward Identities for the two cases and found that they failed for the HV definition but were satisfied for the naive assumption. They conclude from this that Eq.2 "does not appear to be a practicable $\gamma_{5}$-prescription in amplitudes with open fermion legs as it produces a number of spurious anomalies already at the one-loop level." In fact, application of the procedure presented in Collins' book, [5] generalized to include several generations of quarks, leads to the same result as does anticommuting $\gamma_{5}$. The key ingredient to be added is operator mixing under renormalization: flavor diagonal neutral currents can — and do - mix with flavor off-diagonal neutral currents. This is worked through explicitly in the Section 2.2. We draw attention to this here to dispel any doubts that this may have caused regarding the fundamental soundess of Eq.2. On the other hand, it is undeniable that the naive rules lead much more easily to the correct answer.

The objective of this paper is to provide a simple way of obtaining the correct results when $\gamma_{5}$ rears its ugly head by a modification of the regulator-renormalization procedure. These modifications will be derived starting from Eq.2 and, through a series of finite renormalizations, result in rules that are simple to state, that do not involve non-existent objects and do not require non-standard mathematics. The regulating procedure will be changed in such a way that no spurious anomalies arise and so that renormalization by minimal subtraction gives consistent results. Happily for most instances this leads to the naive rules in wide use. Necessarily, closed loops with an odd number of $\gamma_{5}$ 's require a different procedure. The one we have obtained we believe is new, simple to state and to use.

In Section 2 we will review and extend the results of [6] for non-singlet axial vector 
currents, including flavor changing currents and operator mixing. This should make clear the issues and procedures. Section 3 will deal with closed loops and the application to singlet axial vector currents. Another source of spurious anomalies will be identified and dealt with, in which a closed loop is connected to another fermion line. A brief summary of the procedures is given in Section 4. Some details are relegated to the Appendix

\section{Open Lines}

\subsection{Non-singlet axial vector current}

The non-singlet axial current vertex is a good starting point for the discussion of the method. It avoids the complexities of closed loops with an odd number of $\gamma_{5}$ 's while at the same time providing a physically important illustration of the issues.

The problems involved here all hinge on the following points: (1) in calculating an open fermion line containing a $\gamma_{5}$ in one loop or higher order one invariably meets the combinations $\gamma_{\alpha} \gamma_{5} \gamma^{\alpha}$ or $\gamma_{\alpha} \gamma_{\lambda} \gamma_{5} \gamma^{\alpha}$ where a sum over $\alpha$ is implied. If the naive commutation relations were valid, the $\gamma_{5}$ could be pulled out to the left or right and the sum would be determined by vector vertex sum; this can be simply carried to an arbitrary number of contractions such as one meets in multi-loop graphs. However Eq.2 gives a different result:

$$
\begin{array}{r}
\gamma_{\alpha} \gamma_{5} \gamma^{\alpha}=-4 \gamma_{5}+(d-4) \gamma_{5} \\
\gamma_{\alpha} \gamma_{\lambda} \gamma_{5} \gamma^{\alpha}=2 \gamma_{\lambda} \gamma_{5}-(d-4) \gamma_{\lambda} \gamma_{5}
\end{array}
$$

There are two things to note about these relations: first, the only difference from the naive case is the sign of the terms carrying the $(d-4)$ factor, where $d$ is the dimension of spacetime; second, the correction term is twice as big, relatively, for the second equation as it is for the first. Both of these differences will play havoc with Ward identities, which are algebraically valid for the naive commutation relations. These differences will be compounded by the multiple contractions that occur in higher order; indeed, two contractions are required already in one loop, Fig.1. (2) when these relations are encountered in a divergent graph or sub-graph the poles that are produced at $d=4$ by the momentum integrations combine with the $(d-4)$ factors above to produce a constant difference between the two commutation 
relations proportional to $\gamma_{5}$ and $\gamma_{\lambda} \gamma_{5}$, respectively.

At the one loop level, these differences are constants and so can be viewed as a simple finite renormalization. If one used some kind of mass-shell or momentum subtraction, these would be absorbed into the infinite renormalization constants and never seen again. If, on the other hand one simply subtracts off the pole at $d=4$, possibly along with some fixed constants, the difference between the two would remain in the nominal renormalized result. (An extra trap occurs in the case of the Landau gauge which gives a finite result and does not force a renormalization.) This of course cannot be and the finite renormalization must be done if the axial vector current is to obey the Ward identities required of it.

This result is very suggestive that the full axial vector vertex function, to all orders in QCD calculated according to Eq.2, is a finite renormalization of that calculated naively. Some time ago we reported in [6] results directed at clearing up a problem of the generic type we are discussing here, [14. An inductive proof was given that, to all orders in QCD for massless quarks

$$
\Gamma_{\mu}^{5}=z\left(\alpha_{s}\right) \Gamma_{\mu} \gamma_{5}
$$

Here $\Gamma_{\mu}$ and $\Gamma_{\mu}^{5}$ denote, respectively, the vector and axial vector current vertices calculated using Eq.@ with the standard $d$-dimensional $\gamma$-algebra and with the divergences removed by minimal subtraction. $z\left(\alpha_{s}\right)$ is a finite renormalization constant. Evidently the chiral symmetry which is broken by the rules of Eq.2 can be restored by defining a renormalized axial current which is $1 / z\left(\alpha_{s}\right)$ times the minimally subtracted current. This result can obviously also be obtained by calculating from the beginning with anticommuting $\gamma_{5}$.

Eq.5 is a very explicit way to express the result for the massless theory. We will sketch the extension of this inductive procedure to $m \neq 0$ in order to emphasize the important issues. For any non-singlet vertex graph or subgraph $G$ with the $\gamma_{5}$ attached to an open line and whose external legs are in $d=4$, denote by $\tilde{G}$ the same graph evaluated using the naive commutation relations; viz. one simply anticommutes $\gamma_{5}$ out of the graph naively before doing the gamma algebra. $\gamma_{5}$ can be anticommuted out in either direction, the result is the same.

Let $\Gamma_{\mu}^{5(n)}$ denote the axial vector vertex to $n^{t h}$-order in the QCD coupling $\alpha_{s}$ and 
$\widetilde{\Gamma}_{\mu}^{5(n)}$ the corresponding sum of graphs using the anti-commuting $\gamma_{5}$. Because the term in the lowest order graph, Fig.1, proportional to $m$ is convergent and because of Eq. $n=2$ it is obvious that

$$
\Gamma_{\mu}^{5(2)}=z_{2}\left(\alpha_{s}\right) \widetilde{\Gamma}_{\mu}^{5(2)}
$$

This is used as the first step in the inductive argument based on the Dyson-Schwinger equation illustrated in Fig.2. We do not wish to belabor this standard kind of argument here. We just indicate that in each of the blobs all subgraphs are assumed to be made finite by minimal subtraction. The blob marked $K$ is two-particle irreducible. (One can follow the argument of Bjorken and Drell, [15]. The graphs involving two or three photon intermediate states, which they dispose of by Furry's Theorem or by gauge invariance, do not enter here because this is a non-singlet current.) Thus the $k$ integration is not involved in any divergent subgraphs and the only remaining divergence is the overall integration. So if we differentiate once the sum of graphs represented by Fig.2, see [16], the result will be power-counting convergent and can be evaluated in $d=4$; in particular $k$, the external momentum of the vertex can be taken to be in $d=4$. We then use the induction hypothesis

$$
\Gamma_{\mu}^{5(m)}=z_{m}\left(\alpha_{s}\right) \widetilde{\Gamma}_{\mu}^{5(m)} \text { for } m<n
$$

to replace $\Gamma_{\mu}^{5(m)}$ by its tilded partner, anticommute $\gamma_{5}$ through the $S_{F}$ and $K$ which are now in $d=4$ and conclude that

$$
\begin{gathered}
\Gamma_{\mu}^{5(n)}-z_{n-1}\left(\alpha_{s}\right) \widetilde{\Gamma}_{\mu}^{5(n)}=a_{n} \alpha_{s}^{n} \gamma_{\mu} \gamma_{5} \quad a_{n} \text { a constant, or } \\
\Gamma_{\mu}^{5(n)}=z_{n}\left(\alpha_{s}\right) \widetilde{\Gamma}_{\mu}^{5(n)}
\end{gathered}
$$

This is the desired extension of our earlier result for $m \neq 0$. It is important to emphasize that these results are valid only in the limit that $d \rightarrow 4$ and for the external legs in $d=4$. Otherwise additional "evanescent" operators come in to the relations. See [5]. This caused the derivation to be somewhat fussier than might at first sight seem necessary. It will cause further difficulities in the discussion of closed loops.

This procedure allows us to perturbatively calculate $z\left(\alpha_{s}\right)$ should we wish to. However, it really isn't necessary. Rather we note that exactly the same procedure applied to 
the vertex of the pseudoscalar density yields

$$
\Gamma^{5(n)}=z_{n}^{5}\left(\alpha_{s}\right) \widetilde{\Gamma}^{5(n)}
$$

Note $z_{n} \neq z_{n}^{5}$. But then standard algebra shows that

$$
\left(p^{\prime}-p\right)^{\mu} \widetilde{\Gamma}_{\mu}^{5}\left(p^{\prime}, p\right)=\gamma_{5} S^{-1}(p)+S^{-1}\left(p^{\prime}\right) \gamma_{5}-2 i m \widetilde{\Gamma}^{5}\left(p^{\prime}, p\right)
$$

That is, $\widetilde{\Gamma}_{\mu}^{5}\left(p^{\prime}, p\right)$ is the properly normalized axial vector vertex, and the finite renormalization that is required to remove the spurious anomlies induced by 2 is automatically achieved by using the naive commutation relations. This is, of course, the conventional procedure, but we have justified it here is a way that will be useful later.

One can argue quite generally that this must be the result: the two different regulation schemes can only lead to finite renormalization. In the case at hand the only dimension 3 axial vector operator is the the original current so it must be multiplicatively renormalized. Because the minimal renormalization of $\widetilde{\Gamma}_{\mu}^{5}\left(p^{\prime}, p\right)$ is the same as that of the conserved vector current, it is renormalization invariant. Likewise with the pseudoscalar density. Furthermore we see that the minimal renormalization of $\widetilde{\Gamma}^{5}\left(p^{\prime}, p\right)$ is the same as the vertex of $\bar{\psi} \psi$ and since $m \bar{\psi} \psi$ is renormalization invariant in a mass independent renormalization scheme like minimal subtraction [17], so is $m$ times the renormalized pseudoscalar density. That is, the finite renormalizations of the two operators $j_{\mu}^{5}$ and $j^{5}$ must be precisely those that are obtained by the naive commutation relations in order that Eq.11 be renormalization scale invariant.

\subsection{Higher Order Weak Interactions}

The next most complicated situation arises when $\gamma_{5}$ occurs as an internal vertex on an open line, as it does in higher order weak interaction calculations. We do not propose to discuss the renormalizability of $S U(2)_{L} \otimes U(1)$ here; rather we will examine some important examples in low orders in the weak interactions. A very early study of various $\gamma_{5}$ schemes in weak decays may be found in Marciano [18. 
The simplest and most obvious example of the need to carry out the finite renormalization that is achieved by naive $\gamma_{5}$ commutation rules is the vertex correction to single $W^{ \pm}$ exchange, to which must be added the unphysical charged scalar $\phi$ exchange, Fig.3. Because of the different factors in Eq.3 3 and Eq. 4 if they are used without the finite renormalization the amplitude will contain an unphysical term

$$
C g^{2} \frac{\left(m_{u}+m_{d}\right)\left(m_{s}+m_{c}\right)}{k^{2}-\xi M_{W}^{2}}
$$

where $C$ is a non-zero constant. The finite renormalization constants for the two graphs are different and are such as to compensate for this term. Using naive $\gamma_{5}$ from the beginning avoids the necessity of doing this. This is the simplest example which illustrates that removal of infinities is not sufficient to guarantee the consistency of the theory, and that attention must be paid to the spectrum. [19].

Henceforth in all graphs in this section we will assume that, for QCD corrected vertex graphs and subgraphs, the required finite renormalization has been carried out; i.e. within divergent vertex subgraphs, naive $\gamma_{5}$ is used along with minimal subtraction. If the graphs are overall divergent there will in general be different renormalizations for the two schemes, and this will be our focus here.

Let us start by examining the second order weak process mentioned in the introduction, [13]. Here the electroweak Ward identities will force the choice of $\gamma_{5}$ commutation relations. Consider the set of graphs Fig.4. All of these except for the unphysical $\phi$ graphs are finite by power counting or by the GIM mechanism [20], and so give finite results independent of $\gamma_{5}$ commutation relation. The $\phi$ contribution to wavefunction renormalization is, to this order, also independent of $\gamma_{5}$. All the problem then arises from $\phi$ exchange, the third graph, because it has a $\gamma_{5}$ buried inside a divergent graph. The difference between the two procedures is easy to calculate since it comes only from the divergent part. The result is

$$
\Gamma_{\mu}-\widetilde{\Gamma}_{\mu}=\frac{i}{128 \pi^{2}} \frac{g^{2}}{\cos \theta_{W}} C_{b s} \gamma_{\mu}\left(1-\gamma_{5}\right) / 2
$$

where $C_{b s}=\sum V_{s i}^{\dagger} V_{i b} m_{i}^{2} / M_{W}^{2}$; the sum on $i$ goes over the quarks $u, c$ and $t$. At first sight it is not obvious how to account for this difference because there is no zeroth order flavor 
changing $Z$ coupling to apply a finite renormalization to. The answer is, of course, that under Eq.2 the axial vector current $j_{\mu}^{5}$ is not (partially) conserved. Therefore it is renormalized and mixes with other operators of the same dimension. Indeed, the minimal subtraction is proportional to operators precisely of the form $\bar{b}_{L} \gamma_{\mu} s_{L}$. What is needed here is an additonal finite renormalization proportional to the same operator to restore the chiral symmetry, just as in the first subsection. Thus

$$
j_{\mu}^{5} \rightarrow j_{\mu R}^{5}=j_{\mu}^{5}-Z_{i j}^{d} \bar{d}_{i} \gamma_{\mu} \frac{1-\gamma_{5}}{2} d_{j}-Z_{i j}^{u} \bar{u}_{i} \gamma_{\mu} \frac{1-\gamma_{5}}{2} u_{j}
$$

By imposing $\partial^{\mu} j_{\mu R}^{5}=\Sigma 2 m_{i}^{d} \bar{d}_{i} \gamma_{5} d_{i}+\Sigma 2 m_{i}^{u} \bar{u}_{i} \gamma_{5} u_{i}$ one easily finds that

$$
Z_{b s}^{d}=\frac{i}{128 \pi^{2}} \frac{g^{2}}{\cos \theta_{W}} C_{b s}
$$

exactly the factor found by Barroso et al [13] to be needed. When this renormalization in applied $\Gamma_{\mu} \rightarrow \widetilde{\Gamma}_{\mu}$, and so when this formalism is carried through consistently it produces the correct result. There is no denying, however, that care is required and that it is much simpler and correct to apply the naive commutation relations from the beginning.

We turn now to the process $b \rightarrow s+\gamma$, a problem in which the $\gamma_{5}$ question has received much attention, 21]. We focus attention on the Green's function $\left\langle T\left(j_{\mu}(x) b(y) \bar{s}(z)\right\rangle\right.$ to lowest order in the weak coupling $g$ but, eventually, to all orders in $\alpha_{s}$. The simplest graphs shown in Fig. 5 are of order $g^{2}\left(\alpha_{s}\right)^{0}$. The wiggly lines refer to either the $W$ boson or to its unphysical charged scalar partner. (Note that the correct hermitian axial vector vertex is $\left[\gamma_{\alpha}, \gamma_{5}\right] / 2$.) These graphs give the same result for the vertex renormalization in either scheme because GIM [20] makes the $W$ contribution finite and $\gamma_{5}$ appears only external to the divergent loop in the $\phi$ contribution in (a) and (b). The $W$ contribution to (c) and (d) leads to an off-diagonal mass renormalization which cancels between the two when the external legs are on shell. It is well-known that such terms can be removed by a linear redefinition of the fields and have no physical consequences [22]. For an explicit realization of the redefinition appropriate to this case see [23]; in general, the left- and right-handed components are 
transformed differently:

$$
\psi \rightarrow \sqrt{Z_{0}} \psi \text { and } \bar{\psi} \rightarrow \bar{\psi} \sqrt{\bar{Z}_{0}}
$$

where $\bar{Z}=\gamma_{0} Z^{\dagger} \gamma_{0}$ and

$$
Z_{0}=Z_{0}^{L} \frac{1-\gamma_{5}}{2}+Z_{0}^{R} \frac{1+\gamma_{5}}{2}
$$

$Z_{0}^{L, R}$ are matrices in flavor space; to the order we are working in $g$ the diagonal elements can be taken to be unity. The transformations are not necessarily real; for that reason the redefinition disposes of off-diagonal mass renormalization as well as wave function renormalization. To this order the renormalization is pure imaginary (corresponding to a unitary transformation which rotates the off diagonal mass terms away).

This case differs from the previous case because the vector current in conserved in either scheme; it does not depend on the $\gamma_{5}$ definition. Therefore we expect that there is no current renormalization and the only change in the Green's function must result from the quark propagator renormalization. We write the Green's function as a matrix in $b$ and $s$ space

$$
\left(S \Gamma_{\mu} S\right)_{b s}=S_{b b} \Gamma_{\mu b s} S_{s s}+S_{b b} \Sigma_{b s} S_{s s} \Gamma_{\mu s s} S_{s s}+S_{b b} \Gamma_{\mu b b} S_{b b} \Sigma_{b s} S_{s s}
$$

When projected on shell this gives $\Gamma_{\mu b s}+\Sigma_{b s} S_{s s} \Gamma_{\mu s s}+\Gamma_{\mu b b} S_{b b} \Sigma_{b s}$ for the matrix element for $b \rightarrow s+\gamma$.

Now go to order $\alpha_{s}$; Fig.6 shows the graphs relevant to our problem. To these must be added graphs related by symmetry, those with $W \rightarrow \phi$, graphs where $W$ and $g$ are disjoint as well as self energy and vertex counter terms. In graphs Fig.6b,g,h and i the $\gamma_{5}$ is anticommuted naively out of the subgraphs to compensate for the finite axial vector matrix renormalization. The mass renormalization obtained in lowest order enters in Fig.6c and its partner (not shown). It is easy to show by partial fractions that this

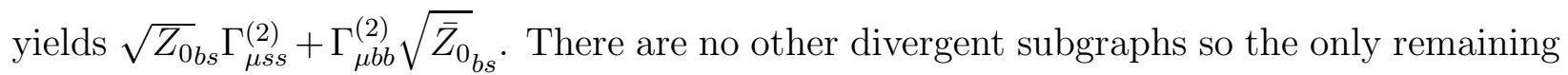
difference can come from the overall divergence of the sum of all the graphs. This has the form $\alpha_{s} g^{2}\left(a_{1} \gamma_{\mu}+a_{1}^{5} \gamma_{\mu} \gamma_{5}\right)$. Gauge invariance ensures that the self energy difference is given by

$$
\alpha_{s} g^{2}\left(-a_{1} \gamma \cdot p-a_{1}^{5} \gamma \cdot p \gamma_{5}+\Delta m_{1}+\Delta m_{1}^{5} \gamma_{5}\right)
$$


Changes in the diagonal Green's functions do not enter the process $b \rightarrow s+\gamma$ at order $g^{2}$. This form is again equivalent to a linear field redefinition at order $g^{2} \alpha_{s}$. (The explicit form can be obtained from the formulas in [23], but we won't need them.) These differences will cancel in the physical amplitudes with $b$ and $s$ quarks on shell and so the two schemes will give the same result; there is no finite renormalization required.

In order to proceed to higher order it is necessary to determine the change in the $g b \bar{s}$ vertex $\Gamma_{\mu}^{g}$ in going from one scheme to the other. One can see explicitly that to this order the change is exactly the same as for the $\gamma b \bar{s}$ vertex. (The set of graphs is similar to Fig. 6 with the omission of $6 \mathrm{a}$ and $6 \mathrm{~b}$ and the addition of graphs containing the triple-gluon vertex.) Using induction, as usual, we assume that to any order $m<n$ that

$$
\begin{aligned}
S^{(m)} & =\sqrt{Z_{m}} \widetilde{S}^{(m)} \sqrt{\bar{Z}_{m}} \\
\Gamma_{\mu}^{(m)} & =\sqrt{1 / \bar{Z}_{m}} \widetilde{\Gamma}_{\mu}^{(m)} \sqrt{1 / Z_{m}} \\
\Gamma_{\mu}^{(m) g} & =\sqrt{1 / \bar{Z}_{m}} \widetilde{\Gamma}_{\mu}^{(m) g} \sqrt{1 / Z_{m}}
\end{aligned}
$$

continuing with matrix notation. Then by the usual differentiation we have that

$$
\begin{gathered}
\Gamma_{\mu}^{(n)}=\sqrt{1 / \bar{Z}_{n-1}} \widetilde{\Gamma}_{\mu}^{(n)} \sqrt{1 / Z_{n-1}}+g^{2} \alpha_{s}^{n}\left(a_{n} \gamma_{\mu}+a_{n}^{5} \gamma_{\mu} \gamma_{5}\right) \\
\Gamma_{\mu}^{(n) g}=\sqrt{1 / \bar{Z}_{n-1}} \widetilde{\Gamma}_{\mu}^{(n) g} \sqrt{1 / Z_{n-1}}+g^{2} \alpha_{s}^{n}\left(a_{n}^{g} \gamma_{\mu}+a_{n}^{g 5} \gamma_{\mu} \gamma_{5}\right) \\
\Sigma^{(n)}(p)=\sqrt{1 / \bar{Z}_{n-1}} \widetilde{\Sigma}^{(n)}(p) \sqrt{1 / Z_{n-1}}+\sum_{m=0}^{n} g^{2} \alpha_{s}^{m}\left(b_{m} \gamma \cdot p+b_{m}^{5} \gamma \cdot p \gamma_{5}+\Delta m_{m}+\Delta m_{m}^{5} \gamma_{5}\right)
\end{gathered}
$$

The terms in the sum for $m<n$ are fixed by the induction hypothesis:

$$
\sum_{m=0}^{n-1} g^{2} \alpha_{s}^{m}\left(b_{m} \gamma \cdot p+b_{m}^{5} \gamma \cdot p \gamma_{5}+\Delta m_{m}+\Delta m_{m}^{5} \gamma_{5}\right)=\left(\gamma \cdot p-m_{s}\right){\sqrt{Z_{n-1}}}_{b s}+{\sqrt{\bar{Z}_{n-1}}}_{b s}\left(\gamma \cdot p-m_{b}\right)
$$

but then we can read off from Eq.25 the terms of order $\alpha_{s}^{n}$ in these renormalization constants. The electromagnetic Ward identity implies that $a_{n}=-b_{n}$ and $a_{n}^{5}=-b_{n}^{5}$ and so to order $n$ the physical matrix element is unchanged. 
We need to also argue that the $g b \bar{s}$ vertex change is the same. Since the change is a constant mattix of the same form as the photon vertex we can fix it by projecting on-shell. Then, because the color current is conserved (or equivalently using BRS identities)

$$
\left(p^{\prime}-p\right)^{\mu} \Gamma_{\mu b s}^{g}+\Sigma_{b s} S_{s s} \Gamma_{\mu s s}^{g}\left(p^{\prime}-p\right)^{\mu}+\left(p^{\prime}-p\right)^{\mu} \Gamma_{\mu b b}^{g} S_{b b} \Sigma_{b s}=0 .
$$

for $\gamma \cdot p^{\prime}=m_{b}$ and $\gamma \cdot p=m_{s}$. This fixes the constants to be the same as in the photon vertex at order $n$.

Thus the induction works and we find, as anticipated, that the only change in the Green's functions results from wave function renormalization. The consequence of this is that the same result will be given by either scheme, to any order in QCD; no finite renormalization enters. This result is not surprising. There was considerable reason to anticipate the result from the many explicit calculations to two loop order for the effective four fermion theories, [21]. It is useful to work it through, however, to show that the form of the difference between the two schemes is as anticipated.

The conclusion is that, since one must treat $\gamma_{5}$ naively within the divergent axial vector vertex subgraphs and since it is so much simpler otherwise and the results are identical—one should certainly use naive $\gamma_{5}$ for all open lines.

The weak corrections to flavor diagonal processes to order $g^{2}$ are more complicated to discuss. First, one must take into account the corrections to the external on shell wavefunctions and show that these will yield the same physical result. This is straightforward. One must also consider the closed fermion loops that arise for the diagonal case. These will be discussed in the next section.

\section{Closed Loops}

In this section, we will examine several classes of closed loop graphs. In all of them the graphs will contain one and only one closed fermion loop to which one or more axial vector or pseudoscalar vertices are attached. The fermions in the loops will always be massive. Arbitrary gluonic corrections to these loops are allowed and, in particular, the $\gamma_{5}$ may appear inside a divergent subgraph. All self-energy and vertex subgraphs are taken to be renor- 
malized by minimal subtraction. Starting, as we are, from the $\gamma_{5}$ definition of 't Hooft and Veltman [1], axial vector and pseudoscalar vertices require additional finite renormalization as discussed in Section 2. The equivalence of this to using naive $\gamma_{5}$ commutation relations that was derived there is valid only when the external fermion legs are in $d=4$, and so the use of that procedure for divergent subgraphs within closed loops must be justified or modified.

The classes we will examine are (1) loops which are superficially convergent, (2) divergent loops corresponding to renormalization parts : AA or PP bubbles and VAA triangle, (3) weak corrections to closed loops, (4) anomalous triangle and box diagrams, and (5) graphs constructed by attaching the closed loop to an open fermion line. Class (4) is very special: consistency of gauge theories require that the triangles within the gauge theory be non-anomalous so the anomaly can occur consistently only for non-gauged, external currents such as interpolating fields for hadronic matrix elements. An important example is the operator corresponding to the longitudinal polarization in deep inelastic lepton-nucleon scattering [24]. Although it is special, it has become a touchstone of any method of treating $\gamma_{5}$ and so cannot be ignored, even though it demands much more careful attention than the other cases. Class (5) represents another potential source of spurious anomalies. Completeness requires that the method be adapted to this class as well.

The broadest class of convergent loops are those connected to more than four external currents and are two and three gluon irreducible. Graphs of this type are superficially convergent and the fermion loop is powercounting convergent as well. Thus, after doing the subgraph subtractions, the corresponding momenta can be taken to be in $d=4$ [5] and any required finite renormalization of axial vector or pseudoscalar vertex subgraphs can be achieved by treating $\gamma_{5}$ naively within the subgraph and, a fortiori, $\gamma_{5}$ can be moved naively around the loop. The only proviso is that $\gamma$-matrices within other divergent subgraphs must be contracted within that subgraph and not across the $\gamma_{5}$. To fail to observe this proviso would run afoul of the inconsistency mentioned in Section 2, and give the incorrect renormalization of that subgraph.

Turning to class (2), let us look at the bubble graphs containing non-singlet $\gamma_{5}$ vertices. There are four of these: each vertex can be either axial vector or pseudoscalar. We denote 
those calculated using the $\mathrm{HV}$ definition by $\Pi_{\mu \nu}^{A A}$ and $\Pi^{P P}$, etc.. Correspondingly we use the notation $\tilde{\Pi}_{\mu \nu}^{A A}$ and $\tilde{\Pi}^{P P}$ for the same quantities calculated using the naive commutation relations; there is no ambiguity in the latter. By differentiating three times with respect to the fermion mass or the external momentum one creates an overall power counting convergent integral so that the results of Section 2 can be used to renormalize the divergent $\gamma_{5}$ subgraphs and we have

$$
\begin{aligned}
& \Pi_{\mu \nu}^{A A}(q)=z^{2} \widetilde{\Pi}_{\mu \nu}^{A A}(q)+T\left(g_{\mu \nu} q^{2}-q_{\mu} q_{\nu}\right)+L q_{\mu} q_{\nu}+M m^{2} g_{\mu \nu} \\
& \Pi_{\mu}^{P A}(q)=z z_{5} \widetilde{\Pi}_{\mu}^{P A}(q)+R q_{\mu} m^{2} \\
& \Pi^{P P}(q)=z_{5}^{2} \widetilde{\Pi}^{P P}(q)+S q^{2}+N m^{2} .
\end{aligned}
$$

In addition to the desired vertex renormalization this procedure generates extra terms, as shown, with $T, L, M, R, S, N$ constants. These terms correspond precisely to the ambiguity of defining the product of local operators at the same point of the form $\partial_{\mu} \partial_{\nu} \delta^{(4)}(x-y)$, $m^{2} g_{\mu \nu} \delta^{(4)}(x-y)$ etc. 25. It is very similar to the ambiguity discovered many years ago by Chanowitz, Furman and Hinchliffe [10]. The resolution of this ambiguity is part of the definition of the axial current. If the bubbles occur inside a graph, as corrections to the weak boson propagator, all of these constants except $T$ must be chosen so that $\Pi_{\mu \nu}^{A A} \rightarrow$ $\widetilde{\Pi}_{\mu \nu}^{A A}, \Pi_{\mu}^{P A} \rightarrow \widetilde{\Pi}_{\mu}^{P A}$ and $\Pi^{P P} \rightarrow \widetilde{\Pi}^{P P}$ in order to avoid unphysical poles in graphs such as in Fig.7. This is the same point made at the beginning of Sec. 2.2. $T$ is fixed by requiring that, for $m=0$ the vertex and axial vector bubbles are the same to preserve chiral symmetry [6].

Next consider the VAA graph of Fig.8a. This is known to be free of true anomalies. However, it is not superficially convergent and so if it is calculated, alternatively, with Eq.2 or with naive $\gamma_{5}$ different results are obtained, the difference being of the form

$$
A\left(p-p^{\prime}\right)_{\lambda} g_{\mu \nu}+B\left\{\left(p+p^{\prime}\right)_{\mu} g_{\lambda \nu}-\left(p+p^{\prime}\right)_{\nu} g_{\mu \lambda}\right\}
$$

where $A$ and $B$ are finite constants. As in the last case, this difference corresponds to the amibiguity in the definition of three local currents at a singlepoint of the form $g_{\mu \nu} \delta^{(4)}(x-y) \partial_{\lambda} \delta^{(4)}(y-z)$ etc. To resolve this ambiguity, consider the interesting case where this arises when each boson is in $S U(2)_{L}$. See Fig.8b. Then by Furry-like arguments 
only the VVV and three VAA triangles survive, proportional to $\epsilon_{a b c}$. Naively all four are equal but by Eq. 31 the three VAA are different from the VVV and and the sum of the differences is equal to

$$
(A+B)\left\{\left(p-p^{\prime}\right)_{\lambda} g_{\mu \nu}+(k-p)_{\nu} g_{\lambda \mu}+\left(p^{\prime}-k\right)_{\mu} g_{\nu \lambda}\right\} .
$$

This is exactly the form of the bare triple-boson vertex and so the difference between the two is just a finite vertex renormalization, analogous to that seen in Section 2. Evidently, again the naive $\gamma_{5}$ leads to the chiral symmetric equality of VVV and VAA as $m \rightarrow 0$. As in the last example, this renormalization must be carried out if these triangle occur internally in order to avoid unphysical poles. This evidently remains true when arbitrary numbers of gluons are attached to the fermion loop. This justifies the folk theorem, that even numbers of $\gamma_{5}$ can be removed from a closed loop by anticommutation; indeed, to avoid the need for finite renormalizations to restore the spuriously broken chiral symmetry, vital to the consistency of the $S U(2)_{L}$ gauge theory, they must be anticommuted naively and removed from the loop.

Let us next consider the weak corrections to closed loops such as shown in Fig.9, again with an arbitrary number of gluon corrections to the loop. The $W$ vertices will yield loops containing 0,1 , or $2 \gamma_{5}$ 's. One can see that the loops with one $\gamma_{5}$ are convergent: as before, for the photon graph the divergence must be proportional to $K \epsilon_{\lambda \mu \nu \alpha}\left(p-p^{\prime}\right)^{\alpha}$, but electromagnetic current conservation requires that $K=0$. Symmetry requires that the gluon graph have the form $f_{a b c} \epsilon_{\lambda \mu \nu \alpha}\left(p_{1}+p_{2}+p_{3}\right)^{\alpha}$, but this is identically zero. Similar arguments, using the same physics, can be presented for those graphs with box subgraphs coupling $W^{+} W^{-}$to $g g$ in the first instance and four gluons in the second.

For loops containing two $\gamma_{5}$ 's Furry's theorem gives zero for the photon graph. The three gluon graph is non-zero but has a divergence proportional to $f_{a b c} V_{\lambda \mu \nu}$, the usual triple boson coupling. This leads simply to a finite renormalization of the strong coupling constant. Thus, when the weak interactions are taken into account the strong coupling will differ by an amount proportional to $g^{2} \alpha_{s}$ in the two schemes. This is a familiar situation and is not aproblem, Of course, one must be sure to use the same scheme in all graphs in order to preserve BRS symmetry, and to note that the numerical value of $\alpha_{s}$ will depend on the scheme as well. 
The AVV case where the axial current is a singlet under the gauge group of the vector currents is historically the most interesting. For many currents of physical interest, the fermion content of the theory is such that when the loops with all the different fermions are summed the result is power-counting convergent [19, 26]. Thus, for these cases the procedures given for convergent loops in the first paragraph of this section apply and there is no true anomaly. We will not dwell on this but turn at once to the case of an anomalous singlet current. It is imperative that the $\gamma_{5}$ procedure deal naturally with this case in order for it to be considered satisfactory. The AVVV case falls into the same category. The long history of this subject begins, of course, with Adler and Bell and Jackiw [27]. More recent papers that are especially pertinent to this subject are [28], 29] and [30] where the graphs of Fig.10 are calculated.

As in all cases we begin with the fermion loops calculated using the original 't HooftVeltman definition of $\gamma_{5}$, the loop integration and the $\gamma$-algebra being done in $d$-dimensions. The spurious anomaly studied in Section 2 arises here in two loop order for the first time and must be removed by the finite renormalization $z\left(\alpha_{s}\right)$, see Fig.10a and 10l. Because the triangle is not power-counting convergent it is not possible to take the loop momenta $k$ in $d=4$ and to take the limit of the (renormalized) axial vector vertex to $d=4$. Therefore one cannot use Eq.9 to automate the required finite renormalization as we did for the open lines. Notice, by the way, that triangles where the axial vector vertex is replaced by a pseudoscalar vertex are power counting convergent and Eq.10 can be used to automate the finite renormalization $z_{5}\left(\alpha_{s}\right)$. Our goal is to modify the regulation so that the spurious anomaly in the axial vector triangle is automatically renormalized away. We will proceed on the basis of two properties of these graphs. The first is that

$$
\lim _{m \rightarrow \infty} \Gamma_{\lambda \mu \nu}^{5}\left(p, p^{\prime}, m\right)=0
$$

This follows from BRS invariance. See the Appendix for an inductive demonstration of this property. The second property is that all the power counting divergent subgraphs which are not compensated by the usual self-energy and vertex minimal subtractions contain the complete fermion loop. Thus, the combination $\Gamma_{\lambda \mu \nu}^{5}\left(p, p^{\prime}, m\right)-\Gamma_{\lambda \mu \nu}^{5}\left(p, p^{\prime}, M\right)$ is power counting convergent. We may then evaluate this combination making use of this property. There are many possible ways to do this. The only essential one is that we may use Eq.9 
to achieve the required finite renormalization of divergent axial vector subgraphs of the fermion loop; the additional changes induced by this replacement are independent of $m$ and will cancel in the above combination. Beyond that, for example, one could evaluate the graphs using naive $\gamma_{5}$; there would result ambiguities in each term in the sum depending on how the various $\gamma$ 's that appear in the loop are contracted with respect to the $\gamma_{5}$ but these ambiguities will cancel in the sum: because the derivative of these graphs with respect to $m$ is convergent the ambiguities are independent of $m$. We will return to this flexibility later. Thus we have

$\Gamma_{\lambda \mu \nu}^{5}\left(p, p^{\prime}, m\right)=\lim _{M \rightarrow \infty}\left(\Gamma_{\lambda \mu \nu}^{5}\left(p, p^{\prime}, m\right)-\Gamma_{\lambda \mu \nu}^{5}\left(p, p^{\prime}, M\right)\right)=z \lim _{M \rightarrow \infty}\left(\widetilde{\Gamma}_{\lambda \mu \nu}^{5}\left(p, p^{\prime}, m\right)-\widetilde{\Gamma}_{\lambda \mu \nu}^{5}\left(p, p^{\prime}, M\right)\right)$

It is important to bear in mind that $\lim _{M \rightarrow \infty} \widetilde{\Gamma}_{\lambda \mu \nu}^{5}\left(p, p^{\prime}, M\right)$ exists only for the gauge invariant sums - that is, there will be logs of $M$-and not graph-by-graph. It will generate the set of graphs arising from the gauge non-invariant vertices $Z_{A}\left(\alpha_{s}\right) \epsilon_{\lambda \mu \nu \alpha} A^{\mu a} \partial_{\alpha} A^{\nu a}$ and $Z_{D}\left(\alpha_{s}\right) \epsilon_{\lambda \mu \nu \rho} f_{a b c} A^{\mu a} A^{\nu b} A^{\rho c}$, which will automatically be the set of counter terms required to restore gauge invariance to the sum in Eq.34. Because the loops that contain a pseudoscalar vertex are powercounting convergent, we obtain

$$
\begin{aligned}
z^{-1} i P^{\lambda} \Gamma_{\lambda \mu \nu}^{5}\left(p, p^{\prime}, m\right) & =\lim _{M \rightarrow \infty} P^{\lambda}\left(\widetilde{\Gamma}_{\lambda \mu \nu}^{5}\left(p, p^{\prime}, m\right)-\widetilde{\Gamma}_{\lambda \mu \nu}^{5}\left(p, p^{\prime}, M\right)\right) \\
& =2 m \widetilde{\Gamma}_{\mu \nu}^{5}\left(p, p^{\prime}, m\right)+A\left(\alpha_{s}\right) \Gamma_{\mu \nu}^{F \tilde{F}}\left(p, p^{\prime}\right) \\
& =2 m z_{5}^{-1} \Gamma_{\mu \nu}^{5}\left(p, p^{\prime}, m\right)+A\left(\alpha_{s}\right) \Gamma_{\mu \nu}^{F \tilde{F}}\left(p, p^{\prime}\right)
\end{aligned}
$$

where $P=p+p^{\prime}$ and

$$
A\left(\alpha_{s}\right) \Gamma_{\mu \nu}^{F \tilde{F}}\left(p, p^{\prime}\right)=-\lim _{M \rightarrow \infty} 2 M \widetilde{\Gamma}_{\mu \nu}^{5}\left(p, p^{\prime}, M\right)
$$

is the true anomaly. $\Gamma_{\mu \nu}^{F \tilde{F}}$ denotes the $F^{a} \widetilde{F}^{a}$ fermion-anti-fermion vertex function [31]. This method constructively determines the coefficient $A\left(g^{2}\right)$. Note that we do not use or address the Adler-Bardeen theorem here [32].

The precise definition of $\widetilde{\Gamma}$ has been left free so far. Any ambiguity is removed in the difference taken in Eq.34. One can choose to do more-or-less as one pleases; the only essential 
is that the $\gamma_{5}$ within the divergent axial subgraph be treated naively as in Eq.9. It is good to make use of this freedom to split the Green's functions into two pieces: one which obeys the naive chiral Ward identity, without any anomaly, and one piece which is "pure" anomaly; i.e. it is the corresponding Green's function of the counter terms $Z_{A}\left(\alpha_{s}\right) \epsilon_{\lambda \mu \nu \alpha} A^{\mu a} \partial_{\alpha} A^{\nu a}$ and $Z_{D}\left(\alpha_{s}\right) \epsilon_{\lambda \mu \nu \rho} f_{a b c} A^{\mu a} A^{\nu b} A^{\rho c}$, as we will now see.

When a closed loop, even a convergent one, is attached to an open fermion line the potential for a new source of spurious anomalies is encountered which cannot be dealt with by simply by modifying $\gamma_{5}$ commutation rules. These are anomalies for which the analog of Eq.37 are violated so that the underlying renormalized operators do not satisfy the anomalous chiral Ward identity

$$
\partial^{\mu} j_{\mu}^{5}=2 m j^{5}+A\left(\alpha_{s}\right) F^{a} \widetilde{F}^{a}
$$

without further finite renormalization of $j_{\lambda}^{5}$, the analog of the non-singlet renormalization in Section 2.1. They occur because, even for convergent loops, the analogs of the relation Eq.37 are valid only for $p, p^{\prime}$ in 4 -dimensions and in the limit $d \rightarrow 4$; corrections proportional to $d-4$ can give a finite result when combined with the overall divergence of the graph. The simplest example is shown in Fig.11 and it occurs already for Abelian theories: to the minimally subtracted graph (a) must be added the finite renormalization of order $\alpha_{s}^{2}$ (b) appropiately chosen in order that the divergence yield graph (c). This was recognized and explicitly calculated by Larin [33] and used to calculate $\alpha_{s}^{2}$ corrections to the Ellis-Jaffe sum rule, 24]. He calculated the required finite renormalization constant by insisting that the renormalized operators satisfy this equation.

Another approach is to modify the regulation procedure in a way analogous to Section 2, so that Eq.39 is automatically satisfied with all operators renormalized by minimal subtractions. There are probably a variety of ways of doing this which could, in principle, in principle generate finite renormalizations to both $j_{\mu}^{5}$ and $F \tilde{F}$. We propose the following way, which seems simple to state and to use. To any order, the complete gauge invariant set of graphs $\Gamma$ breaks up into sets, labeled by index $\{i\}$; each graph in the set have exactly the same gluon configuration and the set is defined by all possible insertions of the axial vector vertex onto the loop. (For example, Fig.10 the sets are (a,b,c,d), (e,f), (g,h,i),(j) and (k).) 
Were it not for the divergences, these sets would individually satisfy the naive chiral Ward identity. For each set, choose a fixed vector vertex - say the marked index in Fig.10 - and naively anti-commute $\gamma_{5}$ from the axial vector vertex to that fixed vertex. By using the convergent combination Eq.34, one does not change $\Gamma_{\lambda \mu \nu}^{5}\left(p, p^{\prime}, m\right)$ except for multiplying by a finite factor analogous to $z$ for the vector vertex chosen. We won't need to calculate that factor, though one could, and that would provide an alternative to the procedure we will describe. Call the function so defined $\Gamma^{N}$, because it manifestly satifies the naive chiral Ward identity even for $d \neq 4$ and for the external momenta with components outside the first four dimensions. This is very important because now this decomposition can be used when this Green's function appears as a subgraph of a divergent graph. The limit $M \rightarrow \infty$ is to be taken before this is attached to an open fermion line, because it is being used to generate the two- and three-gluon counter- terms. The limit does not exist for graphs with an open fermion line 31, 34].

This is the decomposition that we sought and one could just use it with the HV $\gamma_{5}$ calculating the new finite vector renormalization constant, but we would like to bring it more into the spirit of what we have been doing. Separate the primary loop from the rest of the graph by cutting all gluon lines which connect it to the external vector lines. Call this function $L_{\lambda \ldots}^{N}(P, q, \cdots, m)$ where $P$ is the momentum exiting through the axial vector vertex, $q, \cdots$ stands for the momenta of the various gluons which connect the loop to the rest of the graph and $m$ is the fermion mass in the loop. Evidently for each set $i$

$$
i P^{\lambda} L_{\lambda \cdots}^{N}(P, q, \cdots, m)=2 m L_{\ldots}^{N}(P, q, \cdots, m)
$$

implies that

$$
L_{\lambda \cdots}^{N}(0, q, \cdots, 0)=0
$$

where the various other momenta $q, \cdots$ are arbitrary non-vanishing vectors in $d$-dimensions. In particular for the two-gluon function $p+p^{\prime}=P=0$ but $p^{\prime}-p \neq 0$. By forming

$$
L_{\lambda \cdots}^{N}(P, q, \cdots, m)=L_{\lambda \cdots}^{N}(P, q, \cdots, m)-L_{\lambda \cdots}^{N}(0, q, \cdots, 0)
$$

we have created a manifestly power-counting convergent form for $L^{N}$ and all the graphs into which it falls will also be power-counting convergent. We may therefore evaluate these sets 
of graphs in $d=4$, including the fermion loop. Therefore naive $\gamma_{5}$ commutations can - and should-be used to avoid axial vector and pseudoscalar subgraph renormalizations, always bearing in mind the proviso mentioned earlier regarding the divergent self-energy and vector vertex subgraphs of convergent loops.

This gives us the first piece of our decomposition; the other piece, the purely anomalous piece is calculated by taking the limit $M \rightarrow \infty$ of the piece just calculated. As emphasized earlier, for the complete, gauge invariant set the limit exists and is finite. (These steps can be followed through explicitly in the simplest non-trivial example shown in Fig.10 by using the Tables in Akhoury and Titard [29.)

In all of these cases the primary closed loop can be evaluated in $d=4$, after doing the divergent vertex and self-energy insertions, and so $\gamma_{5}$ can be treated naively throughout, especially within the divergent axial vector vertex subgraph. By bringing $\gamma_{5}$ back to where it started, in both the axial vector and the pseudoscalar triangle, the finite vector renormalization we encountered in the course of the derivation is undone. Indeed, all the steps involved there were just to show that this works; they can now be forgotten and we can calculate the Green's functions in the form we started from, provided we make the additive corrections Eq.42 and Eq.34. This "solves" the $\gamma_{5}$ problem for closed loops. To achieve this it has been very important to contrive that $\gamma_{5}$ appears only in convergent closed fermion loop integrals. Other tricks might be used, but the pitfalls are many.

If the function is attached to an open line, because the graph so formed is log divergent, replacing the original graph by this decomposition is equivalent to a finite renormalization of $\Gamma_{\lambda}^{5}$. This is exactly the finite renormalization (e.g. $a_{2}^{\prime}$ of Fig.11, the finite renormalization calculated by Larin [33]) required to maintain Eq.39: the first piece is completely convergent and so gives the first term of Eq.39, and $P$ contracted into the second piece is explictly equal to the anomaly and so minimal subtraction of each maintains that relation.

Because of the form of this decomposition, there should be no difficulty in extending it to graphs with additional closed fermion loops, bearing in mind that $M$ as used above is not a global regulator, but is a tool for determining the non-gauge invariant renormalization constants required. For example, consider the graphs in Fig.12 which are a sample of the 
simplest gauge invariant set of two fermion loop graphs. It is simple to show that $\lim _{M \rightarrow \infty}$ of the sum of all these graphs vanishes. There are two finite renormalizations required when using Eq.22: Fig.12c requires a finite renormalization because of the divergent axial vector vertex and Fig.12a requires one for the reasons we have just seen. They can both be avoided by using our technique of separating the subgraphs into two pieces. Note that the appropriate counter-terms for the fermion loops in Fig.12a and 12b are determined by taking the $\lim _{M \rightarrow \infty}$ of those loops before the final pair of integrations are done. Because the graphs are not overall power counting convergent making these replacements for the subgraphs will lead to a result that can differ from our starting point by a constant. Just as before this constant can be determined by the $\lim _{M \rightarrow \infty}$ of the full graphs calculated in this manner.

This method for graphs involving anomalous closed loops is reasonably simple to state and it seems reasonably direct to implement, but only experience will show if it is more efficient than the completely equivalent method of using Eq.2 and calculating the various finite renormalization constants which are needed to compensate for the spurious anomalies introduced thereby. Our principal goal here has been to address a broad range of $\gamma_{5}$ problems and reduce them to a coherent approach within the minimal subtraction approach to renormalization which avoids the algebraic complications of the non-covariant Eq.2 and the necessity (and dangers) of additional finite renormalizations.

The conclusion is that, with the proper care with regard to cases (4) and (5), the HV scheme with the finite renormalizations required by the symmetries of the theory yields results which are equal to those obtained by naive commutation relations.

\section{Summary}

Starting on the basis of the consistency of the definition of $\gamma_{5}$ given in the original discussion of dimensional regularization by 't Hooft and Veltman [1]

$$
\gamma_{5}=i \gamma_{0} \gamma_{1} \gamma_{2} \gamma_{3}
$$

and the necessity of making a series of finite renormalizations to remove the spurious anomalies in the chiral symmetry equations that arise from this definition, we have developed a procedure for evaluating Feynman graphs that contain $\gamma_{5}$ and using dimensional regularization 
with minimal subtraction that is simple and avoids the need for the finite renormalizations. The procedure is both simple to state and simple to implement:

(1) For open fermion lines containing axial vector or pseudoscalar vertices, one should calculate the subgraphs containing the $\gamma_{5}$ as if $\gamma_{5}$ anticommutes with all $\gamma$ matrices, i.e. "naive" $\gamma_{5}$. The $\gamma_{5}$ may then be freely anticommuted along the fermion line. This will ensure, without further renormalization, that non-singlet currents satisfy

$$
\left(p^{\prime}-p\right)^{\mu} \Gamma_{\mu}^{5}\left(p^{\prime}, p\right)=\gamma_{5} S_{F}^{-1}(p)+S_{F}^{-1}\left(p^{\prime}\right) \gamma_{5}-2 i m \Gamma^{5}\left(p^{\prime}, p\right)
$$

where all quantities are renormalized by minimal subtraction. This is, of course, the commonly used procedure for this type of graph. We emphasize that here it is derived from the consistent starting point stated at the start of this section and is not offered as a fundamentally alternative procedure.

(2) If $\gamma_{5}$ occurs within a convergent loop in a convergent graph it should be treated as if it satisfies the naive anticommutation relations. This ensures that the renormalizations that are required for divergent axial vector or pseudoscalar vertex subgraphs that may occur within the convergent loop are properly accounted for. The only caveat is that all the $\gamma$-algebra contractions within other divergent subgraphs - self-energy and vector verticesmust be done internally to that subgraph and not contract across the $\gamma_{5}$. The same is true for divergent loops - generalized bubble, triangle or box graphs - which contain an even number of $\gamma_{5}$ 's; this takes account of the overall renormalization required in addition to restore the chiral symmetry between vector and axial vector Green's functions and to ensure that the unphysical gauge dependent poles in vector boson propagators properly cancel against those of the corresponding unphysical scalars.

(3) Superficially divergent loops containing a single axial vector vertex require special attention. We consider only color singlet axial vector currents so the potentially problematic cases are the triangle and box graphs with all their radiative corrections. For definiteness, we consider the other currents to be colored vector currents. Other cases are simpler and easily deduced from this result. If when the various fermions which can circle in the loops are added together the resulting sum is convergent, as it is for the electroweak axial vector current in the standard model of fermion doublets, then as in (2), $\gamma_{5}$ should be treated naively. 
The interesting case is when the sum is not power-counting convergent and a true anomaly occurs. There are many ways to proceed; one can define a unique way by insisting on writing the gauge invariant set as a sum of a term which obeys the naive chiral Ward identity and a term which is pure anomaly. This can be achieved in the following way: for any graph with a single fermion loop attached to the axial vector current and any number of gluons, subtract from it the same graph with everything the same except that in the fermion loop the total momentum entering the axial vector vertex $P=0$ and the mass of the fermion is set to zero. For that combination, every subgraph, including the whole graph, containing the fermion loop is superficially convergent. Therefore it can be calculated using naive $\gamma_{5}$. Furthermore, it satisfies the naive chiral Ward identity. The complete, gauge invariant result is obtained by summing all terms of a given order of the above combination and subtracting from it the finite function obtained by taking the limit $M \rightarrow \infty$. This generates the complete set of graphs with the vertices $Z_{A}\left(\alpha_{s}\right) \epsilon_{\lambda \mu \nu \alpha} A^{\mu a} \partial_{\alpha} A^{\nu a}$ and $Z_{D}\left(\alpha_{s}\right) \epsilon_{\lambda \mu \nu \rho} f_{a b c} A^{\mu a} A^{\nu b} A^{\rho c}$ required as counter-terms to restore gauge invariance.

(4) When a closed loop, convergent or divergent, containing a $\gamma_{5}$ is attached to an open fermion line creating a graph which is overall divergent there is a new source for potential spurious anomalies in the sense that the anomalous chiral Ward identity arising in case (3)

$$
\partial^{\mu} j_{\mu}^{5}=2 m j^{5}+A\left(\alpha_{s}\right) F^{a} \widetilde{F}^{a}
$$

is not preserved by minimal subtraction and an additional finite renormalization of $j_{\mu}^{5}$ is required. Use of the above decomposition avoids this problem because the convergent combination, since it satisfies the naive chiral Ward identity, has good asymptotic behaviour in the integration momenta and so for this term the potentially divergent graphs are in fact power-counting convergent and contraction with $P$ gives just the first term in Eq.45. The remaining term is precisely the anomaly so that the minimal subtraction of those terms is identical to minimal subtraction of the anomaly-two fermion vertex.

These procedures have been derived to all orders in QCD and to second order in the electroweak axial coupling for graphs with an open fermion line, a single closed fermion loop and a closed loop connected to an open fermion line. There should be no problem in extending this to graphs with additional fermion loops. We are not aware of any problem in going to higher order in the electroweak coupling, but we have not examined it in detail. 
Acknowlegements: Thanks are due to W. Marciano, R. Akhoury, J. Collins, R. Kauffman, J. Korner, A.Soni for helpful comments and special thanks are due to Eduardo deRafael who called my attention to this problem many years ago and to Scott Willenbrock whose persistent questions reawakened my interest much more recently. 


\section{A Appendix}

The following argument is needed in Section 3 and is included here for completeness; it may be well known but we haven't found a source for this specific argument although the general approach follows, as does much of this work Collins' book [5]. We show that a gauge invariant set of graphs for AVV or AVVV containing only the fermion loop to which the axial vector current is attached must vanish as the fermion mass $m$ goes to infinity.

Start with the simple loop, properly symmetrized in the external vector legs. As $m \rightarrow \infty$ this amplitude must go to a dimensionless function of $m / \mu$ times $\epsilon_{\lambda \mu \nu \alpha}\left(p-p^{\prime}\right)^{\alpha}$ or $\epsilon_{\lambda \alpha \beta \gamma}$ for the AVV or AVVV case respectively. The coefficients do not depend on the external momenta because two derivatives of the first case or one of the second with respect to the external momenta must vanish as $m \rightarrow \infty$. BRS symmetry requires that the divergence of the amplitude with respect to one vector index must vanish when it is contracted into the polarization vector of the other on-shell vector legs. These tensors do not have that property and so, because the coefficient of the tensor does not depend on the momenta, it must vanish identically. Evidently, a simple loop with more than three gluon lines attached will vanish by power counting.

For an inductive argument, assume that to some order $n$ it has been shown that a gauge invariant sum of graphs with two or three gluon legs and one axial vector current vanish as $m \rightarrow \infty$. To order $n+1$ a subset of the graphs have the external gluons attached directly to the fermion loop. By the above argument these must go to $f(m / \mu) \epsilon_{\lambda \mu \nu \alpha}\left(p-p^{\prime}\right)^{\alpha}$ or $g(m / \mu) \epsilon_{\lambda \alpha \beta \gamma}$ for the AVV or AVVV case respectively. For short, in the following discussion, we will call this "going to a constant". For the remaining graphs, examine any subgraph which contains the fermion loop and has $r$ external legs. If $r>3$ these subgraphs vanish as $m \rightarrow \infty$. For $r=2,3$ add together all the graphs which are obtained by replacing the subgraph in question by another subgraph of the same order and with the same number of external legs. The sum of these subgraphs is gauge invariant and by the induction hypothesis it vanishes as $m \rightarrow \infty$. Subgraphs which have ghost lines connecting the fermion loop to the external gluons are power counting convergent and so vanish as $m \rightarrow \infty$. Therefore only the case where all internal lines of the graph are of order $m$ as $m \rightarrow \infty$ are possibly 
non-vanishing and the sum of this subset of graphs may go to a constant. However, when all the graphs of order $n+1$ are added together the sum of these constants must vanish. Therefore, the induction goes through and the gauge invariant set must vanish as $m \rightarrow \infty$ at any order . 


\section{References}

[1] G.'t Hooft and M.Veltman, Nucl. Phys.B44, 189 (1972).

[2] C.G. Bollini and J.J. Giambiagi, Phys. Lett. B40, 566 (1972), G.M. Cicuta, E. Montaldi, Nuovo Cim. Lett. 4, 329 (1972), J.F. Ashmore, Nuovo Cim. Lett.4, 289 (1972), D.A. Akyeampong and R. Delbourgo, Nuovo Cim. 17A, 578 (1973), T. Marinucci and M. Tonin, Nuovo Cim. 31A, 381 (1976).

[3] G.'t Hooft, Nucl. Phys. B61, 455 (1973).

[4] P.Breitenlohner and D.Maison, Commun. Math. Phys. 52, 11 (1977).

[5] John Collins, Renormalization, Cambridge University Press, Cambridge, 1984.

[6] T.L.Trueman, Phys. Lett.88B, 331, (1979).

[7] Guy Bonneau, Int.Jour.Mod.Phys.5, 3831 (1990).

[8] William Bardeen, in Proceedings XVI International Conference on High Energy Physics (National Accelerator Laboratory, Batavia, IL) (1972) .

[9] W.A. Bardeen, R.Gastmans and B.Lautrup, Nucl.Phys. B46, 319, (1972)

[10] M.Chanowitz, M.Furman, and I.Hinchliffe, Nucl.Phys. B159, 225 (1979).

[11] S.Gottlieb and J.T.Donohue, Phys.Rev.D20, 3378 (1979).

[12] J.G.Korner, D.Kreimer, and K.Schilcher, Z.Phys. C54, 503, (1992), D. Kreimer, UTASPHYS-94-01.

[13] A. Barroso,M.A.Doncheski,H.Grotch,J.G.Korner, and K.Schilcher, Phys. Lett.261, 123 (1991).

[14] O.Nachtmann and W.Wetzel, Phys. Lett. 81B, 211 (1979).

[15] J.D.Bjorken and S.D.Drell, Relativistic Quantum Fields McGraw-Hill, New York, 1959.

[16] William E. Caswell and A.D.Kennedy, Phys. Rev.D 25,392 (1982). 
[17] S.Weinberg, Phys. Rev. D8, 3498 (1973).

[18] W.J.Marciano, Nuc. Phys. B84, 132 (1975).

[19] D.Gross and R.Jackiw, Phys. Rev. D6, 477 (1972), C.Bouchiat, J.Iliopoulos and Ph.Meyer, Phys. Lett.B 38, 519 (1972).

[20] S.L. Glashow, J.Iliopoulos, and L. Maiani, Phys. Rev. D2, 1285 (1970).

[21] R. Grigjanis, P.J.O’Donnell, M.Sutherland and H.Navelet, Phys. Letts.B 237, 252 (1990),B. Grinstein, R. Springer, and M. Wise, Nucl. Phys. B339, 269 (1990), A.J. Buras and P.H. Weisz, Nucl. Phys. B333, 66 (1990), M.J. Dugan and B. Grinstein, Phys. Lett. B 256, 239 (1991), M. Misiak, Phys. Lett.B 269, 161 (1991), M. Ciuchini, E. Franco, G. Martenelli, L. Reina and L. Silvestrini, Phys. Lett. B316, 127 (1993), M. Misiak, Nucl. Phys. B393, 23 (1993),A.J. Buras, M. Jamin, M.E. Lautenbacher and P.H. Weisz, Nucl. Phys B400, 37 (1993), G. Curci and G. Ricciardi, Phys. Rev.D 47, 2991 (1993), M. Misiak, Phys. Lett.B 321, 113 (1994), K.Adel and Y.P. Yao, Phys. Rev. D49, 4945 (1994), M. Ciuchini, E. Franco, G. Martenelli and L. Reina, Nucl. Phys. B415, 403 (1994).

[22] G.Feinberg, P.Kabir and S.Weinberg, Phys.Rev.Letters 3, 527 (1959).

[23] J.M.Soares and A.Barroso, Phys. Rev.D, 39,1973 (1989).

[24] J. Ellis and R. L. Jaffe, Phys.Rev. D9, 1444 (1974).

[25] N.N.Bogoliubov and S.V.Shirkov, Introduction to the Theory of Quantized Fields WileyInterscience, New York, 1959.

[26] H.Georgi and S.Glashow, Phys.Rev.D6, 429 (1972).

[27] S. Adler, Phys. Rev. 177, 2426 (1969), J.S. Bell and R. Jackiw, Nuovo Cimento 51, 47 (1969).

[28] D.R.T. Jones and J.P. Leveille, Nucl. Phys. B206,473, (1982).

[29] R. Akhoury and S. Titard, UM-YH-91-21 (1991). 
[30] M. Bos, Nucl. Phys. B404, 215, (1992).

[31] S. Adler in Lectures in Elementary Particles and Quantum Field Theory, eds. S. Deser et al, MIT, Cambridge, MA (1970).

[32] S. Adler and W. Bardeen, Phys. Rev. 182, 1517 (1969)

[33] S.A. Larin, Phys. Lett. B 303, 113 (1993) and CERN-TH.7208/94

[34] J.C. Collins, F. Wilczek and A. Zee, Phys. Rev. D18, 242 (1978). 


\section{Figure Captions}

Fig.1 The lowest order QCD correction to the axial vector vertex.

Fig.2 The Dyson-Schwinger equation for the non-singlet axial vector vertex.

Fig.3 Illustration of the requirement for finite renormalization of the $W$ and $\phi$ couplings.

Fig.4 The lowest order diagrams for $Z \rightarrow b \bar{s}$.

Fig.5 The lowest order diagrams needed for $b \rightarrow s \gamma$. The wiggly lines represent $W$ or $\phi$ in every graph.

Fig.6 A sample of the relevant two loop contributions to $b \rightarrow s \gamma$. See text.

Fig.7 Illustration of the need for finite renormalization of the bubble graphs coupling $W$ 's and $\phi$ 's.

Fig.8 (a)The general VAA triangle and (b) the non-vanishing triangles coupling three $S U(2)_{L}$ currents.

Fig.9 An order $g^{2}$ contribution to $\gamma g g$ and ggg coupling.

Fig.10 The order $\alpha_{s}^{2}$ contributions to the axial vector coupling to two gluons. The vertex marked with a heavy dot is a possible selected vector vertex. To these graphs must be added those with the two external gluons interchanged to maintain the Bose symmetry.

Fig.11 The simplest graphs illustrating the need for a finite renormalization $a_{2}^{\prime}$ of graphs connecting a closed loop to an open fermion line.

Fig.12 Sample of three loop anomaly graphs containing two fermion loops. The constant $a_{2}^{\prime \prime}$ consists of two pieces: one from the vertex renormalization in (c) and one from the renormalization coming from Fig.11 in (a). 